\title{
Lego clocks: building a clock from parts
}

\author{
Michael Brunner, ${ }^{1,3}$ Mirre J.P. Simons, ${ }^{2}$ and Martha Merrow $^{2,4}$ \\ ${ }^{1}$ University of Heidelberg Biochemistry Center, 69120 Heidelberg, Germany; ${ }^{2}$ Department of Chronobiology, University \\ of Groningen, 9750AA Haren, The Netherlands
}

\begin{abstract}
A new finding opens up speculation that the molecular mechanism of circadian clocks in Synechococcus elongatus is composed of multiple oscillator systems (Kitayama and colleagues, this issue, pp. 1513-1521), as has been described in many eukaryotic clock model systems. However, an alternative intepretation is that the pacemaker mechanism-as previously suggested-lies primarily in the rate of ATP hydrolysis by the clock protein KaiC.
\end{abstract}

Biological clocks create endogenous temporal frameworks that correspond to environmental cycles. Amongst these biological timekeepers, the circadian (circa dies, latin for about a day) clock is the best studied. It sets up activity-on all levels, from gene expression to metabolism to behavior-so that they occur at a predictable, perhaps optimal, time of day with respect to the outside world. The daily environmental cycles are relentless, and they create a selective pressure for almost all life forms on earth, as they face challenges such as changing temperature and humidity, and periodic exposure to damaging UV light.

The circadian clock has been characterized in most of the key model genetic organisms, leading to the accumulation of a mass of information concerning its molecular mechanism. A common feature of all clocks so far described is a transcriptional feedback loop (TTL for trancriptional/translational loop) that uses phylum-specific clock genes. In each case, some clock genes function as activators, and some of their downstream targets feed back to inhibit their own transcription. This has been tuned so that the process occurs about once per 24 $\mathrm{h}$ in the absence of an appropriate stimulus from the environment (a zeitgeber, German for time giver). Thus, in constant conditions, a free-running rhythm is observed about once per day, and in normal cycling conditions, an oscillation occurs exactly once per $24 \mathrm{~h}$ (Merrow et al. 2005).

Despite initial findings showing that mutants in these

[Keywords: Circadian rhythm; cyanobacteria; transcription and translation oscillation; KaiC phosphorylation]

Corresponding authors.

${ }^{3}$ E-MAIL michael.brunner@bzh.uni-heidelberg.de; FAX 49-6221-544769. ${ }^{4}$ E-MAIL m.merrow@rug.nl; FAX 31-50-363-2048.

Article is online at http://www.genesdev.org/cgi/doi/10.1101/gad.1686608. so-called core clock genes are arrythmic, their contribution within the clock has become somewhat unclear due to the accumulation of puzzling results. Their arhythmicity is condition-dependent (in constant light, some of these become rhythmic) and is not comprehensive (there can be other $\sim 24-h$ oscillations that continue) (LakinThomas and Brody 2000; Steinlechner et al. 2002; Correa et al. 2003). Although the free-running rhythm is disrupted, the special circadian synchronization, called entrainment, may still be intact (Merrow et al. 1999). The constitutive expression of a normally oscillating clock gene or its combination with another mutation can lead to a rescue of the phenotype (Yang and Sehgal 2001; Collins et al. 2005). Perhaps the biggest challenge to the feedback loop hypothesis came from the sole prokaryotic model organism, Synechococcus elongatus, when it was shown that a self-sustained circadian rhythm of protein phosphorylaion could be mimicked in a test tube using just three clock proteins plus ATP (Nakajima et al. 2005). Obviously, in this case, no transcription is necessary for the basic rhythm-generating mechanism. On the basis of this observation it had been assumed that the phosphorylation rhythm is the pacemaker of the circadian clock of cyanobacteria. In this issue of Genes \& Development, the author of this original observation and his team suggest that even without the phosphorylation rhythm, these cells show oscillations in the transcriptional machinery (Kitayama et al. 2008). This could be telling us something about complexity or, alternatively, we might still be chasing the clock mechanism.

\section{The cyanobacterial clock}

Synechococcus are photosynthetic cyanobacteria, sometimes called "pond scum," and they are largely responsible for oxygenation of the environment on earth. The key clock genes in this species are KaiA, KaiB, and KaiC. KaiC is autophosphorylated and features two Walker domains, which bind ATP (and also other nucleotides, with a lower affinity) and thereby set up their hydrolysis, facilitated by two downstream glutamic acid residues (Hayashi et al. 2003). The KaiC monomers form a hexamer structure that depends partially on nucleotide binding, and whose conformation depends on its phosphorylation state (Mori et al. 2007). KaiA promotes the 
autophosphorylation of $\mathrm{KaiC}$; KaiB discourages it, and thus supports dephosphorylation of KaiC. Initially, it was generally thought that these molecules, through their self-(transcriptionally) regulated expression, created an 24 -h oscillation in all of these features and activities, but the "clock in a tube" indicates that a self-sustained circadian oscillation is encoded in the enzymatic activities of kinase, phosphatase, and ATPase that are part of the proteins (Terauchi et al. 2007). Given that phosphorylation is a cricital aspect of all known circadian clocks (Brunner and Schafmeier 2006), it was this molecular manifestation of rhythmicity-and its consequences on hexamerization of the KaiC proteins-that many of us have been concentrating on.

\section{It's not phosphorylation}

The latest twist in this story has the Kondo laboratory (Kitayama et al. 2008) showing that the circadian KaiC phosphorylation cycle is not strictly required for selfsustained, circadian rhythms. Kitayama et al. (2008) constructed a strain that overexpresses KaiA, the partner that promotes autophosphorylation of KaiC. KaiC was thus arrested in the phosphorylated state. Despite holding this aspect of the clock constant, KaiB and KaiC protein and transcript levels oscillated with a circadian period that was temperature-compensated between $25^{\circ} \mathrm{C}$ and $40^{\circ} \mathrm{C}$. When KaiA overexpression was faclitated in KaiC mutants, they persisted with their characteristic mutant phenotypes. A survey of selected promoters maintained their relative temporal profiles when KaiA was overexpressed.

These data demonstrated that an obvious KaiC phosphorylation cycle is not essential for free-running, temperature-compensated, circadian transcription of cyanobacteria (Kitayama et al. 2008). These three characteristics are fundamental aspects of many circadian clocks; hence, the circadian clock appears to be robust and alive in the cells lacking cyclic phosphorylation of KaiC. With KaiA overexpression, the only defects in circadian properties were a $15 \%$ decrease in period length and a lower amplitude in the transcriptional oscillations. Similarly, the trough levels of KaiC abundance were elevated in KaiA-overexpressing cells, suggesting that the KaiC phosphorylation cycle may enhance the amplitude (peak to trough ratio) of circadian transcription in vivo.

In a second set of experiments, Kitayama et al. (2008) analyzed KaiC phosphorylation mutants in strains with normal levels of KaiA. The mutant S431E;T432E mimics constitutive phosphorylation and has a long $(\sim 48 \mathrm{~h})$ period of $\mathrm{kaiBC}$ promoter activity and KaiC abundance. In a previous study, the Kondo laboratory had shown that the KaiC mutant S431A;T432A was not rhythmic, suggesting that unphosphorylated KaiC might not be able to support circadian rhythmicity (Nishiwaki et al. 2004). However, when a critical lysine residue in the Walker A motif of the C-terminal ATPase domain was replaced by a histidine residue $(\mathrm{K} 294 \mathrm{H})$, this strain displayed a long period rhythm (of PkaiBC-luc) similar to the constitutive phosphorylation mimic mutant (S431E;T432E). However, KaiC-K294H displayed no detectable autokinase activity in vivo or in vitro.

Finally, Kitayama et al. (2008) showed that KaiA-overexpressing cells were arrhythmic at $20^{\circ} \mathrm{C}$, while wildtype cells displayed robust circadian luciferase activity. Similarly, the in vitro rhythm of KaiC phosphorylation was abolished at $20^{\circ} \mathrm{C}$. At elevated temperature, dilution of KaiC by rapidly growing cells may contribute to the amplitude of the KaiC abundance rhythm, and thus partially compensate for the lack of a rhythm of KaiC function produced by the phosphorylation cycle. Conversely, at $20^{\circ} \mathrm{C}$ growth is slow and may not support a KaiC abundance rhythm by growth and dilution, with a consequence that the TTL fails to support circadian rhythmicity. Coupling of these rhythms in cells is suggested by the observation that the trough levels of circadian transcription and KaiC abundance are elevated when the KaiC phosphorylation rhythm is abolished by overexpression of KaiA.

How could the two oscillatory processes contribute amplitude? The global rhythm of gene transcription is a clock-controlled process (Liu et al. 1995) and the Kaibased oscillator supports rhythmic transcription by two pathways (Fig. 1). On one hand, it regulates the activity of a two-component system consisting of the histidine kinase SasA and the response regulator RpaA (Takai et al. 2006). On the other hand, the Kai oscillator supports a circadian rhythm in the compaction-decompaction of

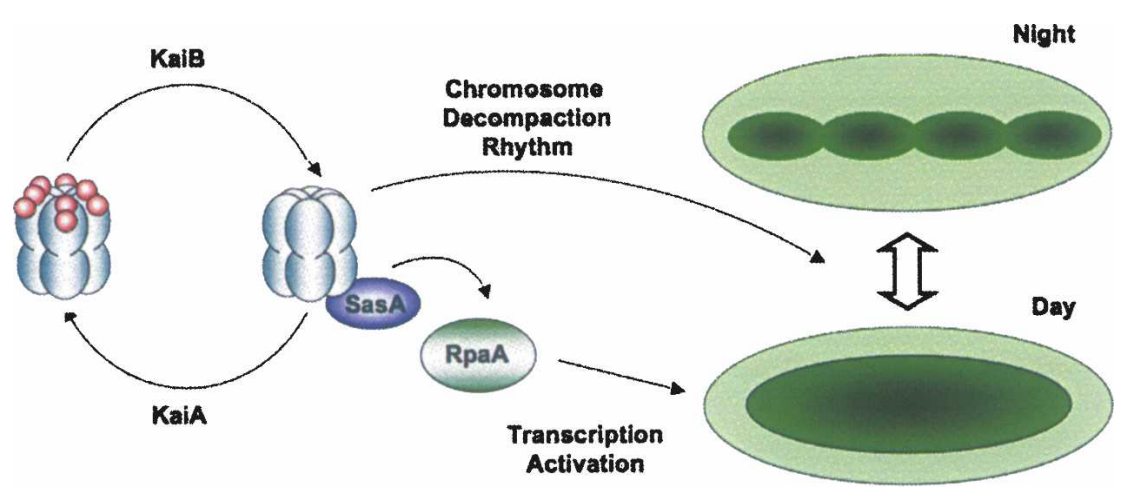

Figure 1. The KaiC hexamer undergoes daily cycles of autophosphorylation (red spheres) and dephosphorylation. Autophosphorylation is stimulated by KaiA during the night; KaiB inhibits KaiA aund supports dephosphorylation of KaiC during the day. Hypophosphorylated KaiC promotes autophosphorylation of the histidine kinase, SasA, which transfers a phosphate to RpaA. Phosphorylated RpaA activates global transciption of genes. The Kai proteins also regulate transcription via daily compaction and decompaction of the cyanobacterial chromosome (dark-green ovals). 
the entire cyanobacterial chromosome (Smith and Williams 2006). In the absence of SasA, rhythmic chromosome compaction is retained, but rhythmic gene expression is attenuated. Thus, decompaction of the chromosome is thought to provide access of the SasA/RpaA system to globally activate transcription (by an as-yetunknown mechanism). It will be interesting to learn how these two pathways are affected when the KaiC phosphorylation rhythm is compromised.

The crucial question arising from the work of Kitayama et al. (2008) is, however, whether the TTL-based KaiC abundance cycle and the KaiC phosphorylation cycle represent two independent self-sustained circadian pacemakers, or whether they reflect the activity of a single oscillator that acts upstream and affects both functions. The former hypothesis is attractive in the light of recent work elaborating mechanisms by which circadian clocks contribute to seasonal behavior through coupling of multiple oscillators (Pittendrigh and Daan 1976; Grima et al. 2004; Stoleru et al. 2004). Several observations, however, favor the latter (single oscillator) hypothesis.

Both KaiC Walker-type domains show ATPase activity, and their binding of ATP-even without hydrolysis (Hayashi et al. 2003) - is required for efficient formation of hexameric rings (Terauchi et al. 2007; Murakami et al. 2008). The rate of ATP hydrolysis by KaiC is more than an order of magnitude higher than its rate of autophosphorylation (Terauchi et al. 2007). In the presence of $\mathrm{KaiA}$ and $\mathrm{KaiB}$, the ATPase activity of KaiC displays a temperature-compensated circadian rhythm that correlates with the circadian phenotype of KaiC mutants. Hence, the ATPase activity of KaiC functions within the self-sustained circadian pacemaker. Autophosphorylation of KaiC inhibits the ATPase, thus forming a negative feedback loop (Fig. 2). This feedback may or may not be part of the pacemaker mechanism. It is therefore of particular interest whether the KaiC phosphorylation mutants described by Kitayama et al. (2008) support an ATPase rhythm in the absence of autophosphorylation of the C-terminal domain. Since KaiC K294H supports a circadian rhythm in vivo, one would predict that ATP hydrolysis by the $\mathrm{N}$-terminal domain is rhythmic without a functional C-terminal ATPase. This inhibition of ATPase activity by KaiC autophosphorylation seems to be required for circadian function, since KaiC S431E;T432E supports a circadian rhythm, while KaiC S431A;T432A does not. Hence, uninhibited hydrolysis of ATP by the C-terminal domain may interfere with a circadian rhythm of ATP hydrolysis (presumably by the N-terminal domain).

Rhythmic ATPase activity of KaiC could have a circadian function independent of the autophosphorylation rhythm (Kitayama et al. 2008). It could produce conformational changes of the hexamer that regulate signaling via the SasA pathway and/or the chomosome compaction pathway. The monomer-hexamer equilibrium of KaiC is regulated by ATP (Terauchi et al. 2007) and levels of KaiC monomer display a circadian rhythm (Mori et al. 2007). Hence, KaiC monomers could have a circadian
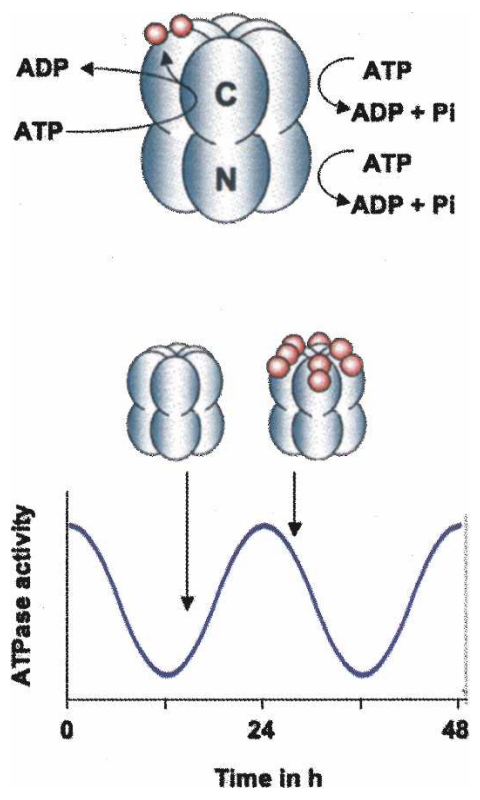

Figure 2. Structure and function of KaiC. (Top) Schematic outline of the KaiC hexamer. The N-terminal (N) and C-terminal (C) domains of a KaiC subunit are indicated. Each domain has low intrinsic ATPase activity and the C-terminal domain has autokinase activity. S431 and T432 in the C-terminal domain are phosphorylated (red spheres) by the C-terminal ATPase of the adjacent KaiC subunit. (Bottom) The ATPase activity and the phosphorylation state of KaiC oscillate with a circadian period. The abundance peaks of nonphosphorylated and phosphorylated KaiC are indicated relative to the ATPase rhythm.

function, potentially even as a signaling kinase. Autophosphorylation of KaiC would then be formally equivalent to an autoinhibition process common in numerous kinase systems (Gietzen and Virshup 1999; Peterson et al. 2000).

\section{Building a clock from pieces}

The detailed knowledge that is accumulating concerning the cyanobacterial clock is complemented by the availability of molecular phylogenetic information on the clock genes. KaiC is the oldest of the three Kai genes at 3.8-3.5 Ga (Dvornyk et al. 2003). In primitive cyanobacteria, in the absence of the modulating partners KaiA and KaiB, which are necessary for a self-sustained circadian rhythm, KaiC could have mediated a light-driven form of gene regulation, using ATP produced rhythmically during the day from photosynthesis to promote hexamerization and to modulate gene expression. Tuning the kinetics of phosphorylation and dephosphorylation could have been a first step away from a driven process toward a temporal structure of gene expression, with hexamers decaying through the night and reforming via a lightdriven response the next day (Fig. 2).

The drive for temporal structure is illustrated in the problem of nitrogen fixation. Oxygen strongly inhibits the nitrogenase enzyme, so, once cyanobacteria had oxy- 
genated the atmosphere, sequestration of nitrogen fixation to nighttime would become favorable (Golden et al. 1997). At around the time that oxygenation occurred, $\mathrm{KaiB}$ and SasA emerged (between 3.5 Ga and 2.3 Ga) (Dvornyk et al. 2003), which may have supported a simple time-specific modulation of transcription until KaiA evolved, allowing anticipation in a full circadian range and forming a self-sustained oscillation.

\section{Putting the pieces together}

It is surprising that, on one hand, we know so much about the cynaobacteria clock, yet, on the other hand, there are many basic circadian properties or clock components that remain undescribed. Most organisms use light as the predominant signal to synchronize to environmental cycles. In cyanobacteria, to date, no light input pathway to the Kai gene clock has been indentified. As noted above, there will be a bolus of ATP produced each day and this could serve to indicate time of day by acting on the KaiC chemistry.

It will therefore be crucial to learn more about the enzymatics of KaiC, in particular about the coupling of the ATPase acitivities of both domains and how the associated changes in the structure and oligomeric state of KaiC are facilitated by the phosphorylation cycle. Finally, little is known about the SasA-independent output of the Kai-based oscillator and the molecular basis of the chomosome compaction rhythm.

Of the many questions still on the table, one of the most obvious is whether the rhythmic ATPase activity is the mechanism underlying the circadian rhythm of transcription in the absence of rhythmic KaiC phosphorylation. The Kondo laboratory is well equipped to attack this question; e.g., by a high-throughput mutant screen using KaiA overexpression strains as a background. The collection of such mutants may center around ATP hydrolysis, revealing ancient and pervasive cellular mechanisms that couple with transcriptional feedbacks to finetune the temporal needs of the host.

\section{Acknowledgments}

Our work is supported by the Dutch Science Foundation (the NWO), The Hersenen Stichting, the German Science Foundation (the DFG), EUCLOCK, a 6th Framework Programme of the European Union, the Fonds der Chemischen Industrie (FCI), and the Rosalind Franklin Fellowship Program of the University of Groningen.

\section{References}

Brunner, M. and Schafmeier, T. 2006. Transcriptional and posttranscriptional regulation of the circadian clock of cyanobacteria and Neurospora. Genes \& Dev. 20: 1061-1074.

Collins, B.H., Dissel, S., Gaten, E., Rosato, E., and Kyriacou, C.P. 2005. Disruption of Cryptochrome partially restores circadian rhythmicity to the arrhythmic period mutant of Dro- sophila. Proc. Natl. Acad. Sci. 102: 19021-19026.

Correa, A., Lewis, Z.A., Greene, A.V., March, I.J., Gomer, R.H., and Bell-Pedersen, D. 2003. Multiple oscillators regulate circadian gene expression in Neurospora. Proc. Natl. Acad. Sci. 100: $13597-13602$.

Dvornyk, V., Vinogradova, O., and Nevo, E. 2003. Origin and evolution of circadian clock genes in prokaryotes. Proc. Nat1. Acad. Sci. 100: 2495-2500.

Gietzen, K.F. and Virshup, D.M. 1999. Identification of inhibitory autophosphorylation sites in casein kinase I $\varepsilon$. J. Biol. Chem. 274: 32063-32070.

Golden, S.S., Ishiura, M., Johnson, C.H., and Kondo, T. 1997. Cyanobacterial circadian rhythms. Annu. Rev. Plant Physiol. Plant Mol. Biol. 48: 327-354.

Grima, B., Chélot, E., Xia, R., and Rouyer, F. 2004. Morning and evening peaks of activity rely on different clock neurons of the Drosophila brain. Nature 431: 869-873.

Hayashi, F., Suzuki, H., Iwase, R., Uzumaki, T., Miyake, A., Shen, J.R., Imada, K., Furukawa, Y., Yonekura, K., Namba, K., et al. 2003. ATP-induced hexameric ring structure of the cyanobacterial circadian clock protein KaiC. Genes Cells 8: 287-296.

Kitayama, Y., Nishiwaki, T., Terauchi, K., and Kondo, T. 2008. Dual KaiC-based oscillations constitute the circadian system of cyanobacteria. Genes \& Dev. (this issue) doi: 10.1101/ gad.1661808.

Lakin-Thomas, P.L. and Brody, S. 2000. Circadian rhythms in Neurospora crassa: Lipid deficiencies restore robust rhythmicity to null frequency and white-collar mutants. Proc. Natl. Acad. Sci. 97: 256-261.

Liu, Y., Tsinoremas, N.F., Johnson, C.H., Lebedeva, N.V., Golden, S.S., Ishiura, M., and Kondo, T. 1995. Circadian orchestration of gene expression in cyanobacteria. Genes \& Dev. 9: 1469-1478.

Merrow, M., Brunner, M., and Roenneberg, T. 1999. Assignment of circadian function for the Neurospora clock gene frequency. Nature 399: 584-586.

Merrow, M., Spoelstra, K., and Roenneberg, T. 2005. The circadian cycle: Daily rhythms from behaviour to genes. $E M B O$ Rep. 6: 930-935.

Mori, T., Williams, D.R., Byrne, M.O., Qin, X., Egli, M., McHaourab, H.S., Stewart, P.L., and Johnson, C.H. 2007. Elucidating the ticking of an in vitro circadian clockwork. PLOS Biol. 5: e93. doi: 10.1371/journal.pbio.0050093.

Murakami, R., Miyake, A., Iwase, R., Hayashi, F., Uzumaki, T., and Ishiura, M. 2008. ATPase activity and its temperature compensation of the cyanobacterial clock protein KaiC. Genes Cells 13: 387-395.

Nakajima, M., Imai, K., Ito, H., Nishiwaki, T., Murayama, Y., Iwasaki, H., Oyama, T., and Kondo, T. 2005. Reconstitution of circadian oscillation of cyanobacterial KaiC phosphorylation in vitro. Science 308: 414-415.

Nishiwaki, T., Satomi, Y., Nakajima, M., Lee, C., Kiyohara, R., Kageyama, H., Kitayama, Y., Temamoto, M., Yamaguchi, A., Hijikata, A., et al. 2004. Role of KaiC phosphorylation in the circadian clock system of Synechococcus elongatus PCC 7942. Proc. Natl. Acad. Sci. 101: 13927-13932.

Peterson, R.T., Beal, P.A., Comb, M.J., and Schreiber, S.L. 2000. FKBP12-rapamycin-associated protein (FRAP) autophosphorylates at serine 2481 under translationally repressive conditions. J. Biol. Chem. 275: 7416-7423.

Pittendrigh, C.S. and Daan, S. 1976. A functional analysis of circadian pacemakers in nocturnal rodents: V. Pacemaker structure: A clock for all seasons. J. Comp. Physiol. [A] 106: 333-355.

Smith, R.M. and Williams, S.B. 2006. Circadian rhythms in gene 
Brunner et al.

transcription imparted by chromosome compaction in the cyanobacterium Synechococcus elongatus. Proc. Natl. Acad. Sci. 103: 8564-8569.

Steinlechner, S., Jacobmeier, B., Scherbarth, F., Dernbach, H., Kruse, F., and Albrecht, U. 2002. Robust circadian rhythmicity of Per1 and Per2 mutant mice in constant light and dynamics of Per1 and Per2 gene expression under long and short photoperiods. J. Biol. Rhythms 17: 202-209.

Stoleru, D., Peng, Y., Agosto, J., and Rosbash, M. 2004. Coupled oscillators control morning and evening locomotor behaviour of Drosophila. Nature 431: 862-868.

Takai, N., Nakajima, M., Oyama, T., Kito, R., Sugita, C., Sugita, M., Kondo, T., and Iwasaki, H. 2006. A KaiC-associating SasA-RpaA two-component regulatory system as a major circadian timing mediator in cyanobacteria. Proc. Natl. Acad. Sci. 103: 12109-12114.

Terauchi, K., Kitayama, Y., Nishiwaki, T., Miwa, K., Murayama, Y., Oyama, T., and Kondo, T. 2007. ATPase activity of KaiC determines the basic timing for circadian clock of cyanobacteria. Proc. Natl. Acad. Sci. 104: 16377-16381.

Yang, Z. and Sehgal, A. 2001. Role of molecular oscillations in generating behavioral rhythms in Drosophila. Neuron 29: 453-467. 


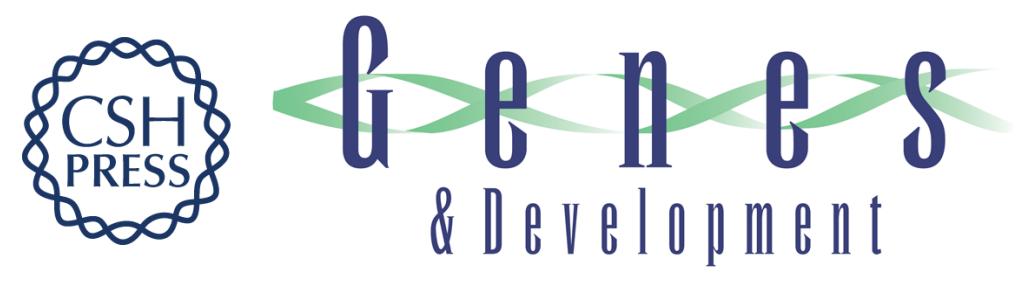

\section{Lego clocks: building a clock from parts}

Michael Brunner, Mirre J.P. Simons and Martha Merrow

Genes Dev. 2008, 22:

Access the most recent version at doi:10.1101/gad.1686608

Related Content Dual KaiC-based oscillations constitute the circadian system of cyanobacteria Yohko Kitayama, Taeko Nishiwaki, Kazuki Terauchi, et al.

Genes Dev. June , 2008 22: 1513-1521

References This article cites 25 articles, 15 of which can be accessed free at: http://genesdev.cshlp.org/content/22/11/1422.full.html\#ref-list-1

Articles cited in:

http://genesdev.cshlp.org/content/22/11/1422.full.html\#related-urls

\section{License}

Email Alerting Receive free email alerts when new articles cite this article - sign up in the box at the top Service right corner of the article or click here.

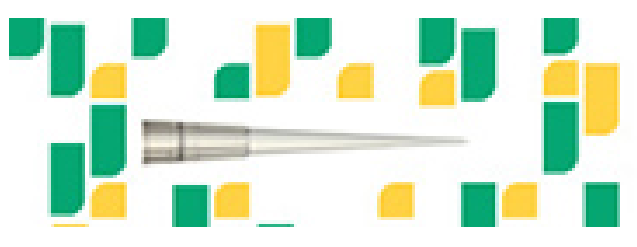

Focused on your science. 